\section{Validação relativa de Questionário de Freqüência Alimentar em gestantes usuárias de serviços do Sistema Único de Saúde em dois municípios no Rio Grande do Sul, Brasil}

\section{Validation of a Food Frequency Questionnaire conducted among pregnant women attended by the Brazilian National Health Service, in two municipalities of the State of Rio Grande do Sul, Brazil}

Andressa Giacomello 1

Maria Inês Schmidt 2

Maria Angélica Antunes Nunes 3

Bruce Bartholow Duncan 4

Rafael Marques Soares 5

Patrícia Manzolli 6

Suzi Camey 7

1-6 Programa de Pós-Graduação em Epidemiologia. Universidade Federal do Rio Grande do Sul. Rua Ramiro Barcelos, 2400, $2^{\circ}$ andar. Porto Alegre, RS, Brasil. CEP: 90035-003.

E-mail: andressa.giacomello@terra.com.br

7 Instituto de Matemática. Universidade Federal do Rio Grande do Sul. Porto Alegre, RS, Brasil.

\section{Resumo}

Objetivos: avaliar o desempenho do Questionário de Frequência Alimentar (QFA), desenvolvido por Sichieri e Everhart, em medir o consumo alimentar de gestantes atendidas pelo Sistema Único de Saúde (SUS), comparando as estimativas de consumo de energia e nutrientes com as obtidas por recordatórios alimentares.

Métodos: foram realizadas duas entrevistas com gestantes em atendimento pré-natal em Bento Gonçalves e Porto Alegre, no Rio Grande do Sul, Brasil. Na primeira, foi aplicado o QFA e, depois, os recordatórios alimentares relativos aos dois dias prévios. Na segunda, foram obtidos novos recordatórios. Médias e limites de concordância para as diferenças entre as duas avaliações de ingestão alimentar foram calculadas conforme Bland-Altman.

Resultados: a diferença entre os métodos mostrou que o QFA superestimou o consumo de energia e nutrientes. O coeficiente de correlação ajustado para energia variou de 0,01 (gordura insaturada) a 0,47 (cálcio). Correlações maiores foram observadas para fibras, vitamina $C$, ácido fólico, cálcio e potássio. Em média, 30\% das gestantes foram classificadas no mesmo quartil de consumo.

Conclusões: o QFA pode ser uma ferramenta útil para avaliar consumo de gestantes em estudos epidemiológicos, contanto que sejam realizados esforços para melhorar seu desempenho. É necessário avaliar a influência de características sócio-demográficas e estado de saúde na validade do método.

Palavras-chave Nutrição materna, Consumo de alimentos, Gestantes, Questionário de Freqüencia Alimentar 


\section{Introdução}

$\mathrm{O}$ estado nutricional materno, antes e durante a gestação, é um forte determinante do desfecho da mesma, tanto na saúde da mãe quanto do recémnascido. ${ }^{1}$ A alimentação na gestação deve prover o aporte suficiente de energia e nutrientes, para promover ganho de peso adequado à mãe e ao recém-nascido. ${ }^{2}$

Para detectar deficiências nutricionais em seu estágio inicial, é necessário avaliar a ingestão alimentar. Entre os estudos dessa natureza, realizados em gestantes brasileiras, observa-se o uso de diferentes metodologias, como recordatório alimentar e questionários de freqüência alimentar.3-5

O Questionário de Freqüência Alimentar (QFA) é amplamente utilizado, visto que mede períodos longos de tempo, e sua execução e a análise dos resultados são fáceis e rápidas, quando comparado a registros e recordatórios. Essas vantagens se traduzem em baixo custo, o que é especialmente importante em estudos epidemiológicos.6,7

Para que o QFA se torne uma ferramenta útil, são necessários estudos de validação, que meçam o grau de concordância entre este e outro método consagrado de avaliação dietética. Em virtude da inexistência de um padrão ouro, essa validação é dita "relativa", ou seja, em referência ao recordatório alimentar. Mesmo assim, estudos comparativos dessa natureza contribuem para minimizar erros na avaliação nutricional que poderiam levar a falsas associações entre dieta e doença. 8,9

O presente estudo teve como objetivos: a) avaliar o desempenho (validação) do QFA, desenvolvido por Sichieri e Everhart, 10 em medir o consumo alimentar de gestantes usuárias do Sistema Único de Saúde de Porto Alegre e Bento Gonçalves; b) verificar a diferença do QFA na estimativa do consumo alimentar entre as gestantes atendidas de Bento Gonçalves e Porto Alegre, duas cidades do Rio Grande do Sul.

\section{Métodos}

O presente estudo de validação relativa foi aprovado pelo Comitê de Ética em Pesquisa da Universidade Federal do Rio Grande do Sul, tendo sido realizado em quatro unidades de saúde do Sistema Único de Saúde, duas em Bento Gonçalves e duas em Porto Alegre, no período de novembro de 2005 a janeiro de 2006. Todas as gestantes que se encontravam nas salas de espera para consulta ou exames de pré-natal foram convidadas para participar da pesquisa. Não foram usados limites de idade ou idade gestacional como critérios de inclusão. Foi excluída no arrolamento apenas uma gestante, portadora de deficiência mental. Após a leitura e esclarecimento do Termo de Consentimento, as gestantes que concordaram participar foram entrevistadas por equipe treinada centralmente. Foram selecionadas 161 gestantes, sendo 85 de Bento Gonçalves e 76 de Porto Alegre, com idades entre 15 e 42 anos.

O questionário de freqüência alimentar desenvolvido por Sichieri e Everhart ${ }^{10}$ foi empregado pela adequação aos objetivos do estudo e por apresentar desempenho satisfatório nos pré-testes. O referido questionário avalia o consumo de alimentos relativos ao último ano e oferece porções padronizadas como opção para avaliar a quantidade consumida nas seguintes freqüências de consumo: mais de três vezes ao dia, duas a três vezes ao dia, uma vez ao dia, cinco a seis vezes por semana, duas a quatro vezes por semana, uma vez por semana, uma a três vezes por mês, nunca ou quase nunca. A lista de alimentos é composta por oitenta alimentos e foi elaborada a partir de dados do Estudo Nacional de Despesa Familiar (ENDEF). 10,11

Nos pré-testes, o questionário foi aplicado a dez gestantes, juntamente com recordatório alimentar de 24hs. Observou-se que o instrumento contemplava a maioria dos alimentos consumidos pelas entrevistadas, sendo necessárias poucas alterações da lista de alimentos. As alterações efetuadas também tiveram por finalidade adequar a lista aos objetivos do estudo. Foram excluídos os itens pão doce, inhame, quiabo e mate, e incluídos arroz integral, macarrão integral e pão integral, pão caseiro e salgadinho. Para o leite, foi dada a opção de integral, desnatado ou semidesnatado, e para suco, refrigerante e iogurte, a opção light/diet ou convencional. Os itens margarina e manteiga foram separados devido às diferenças no conteúdo de colesterol e tipo de gordura.

$\mathrm{Na}$ entrevista inicial foram coletadas informações sócio-demográficas e aplicado o QFA de dois recordatórios alimentares, relativos aos dois dias precedentes. Foi agendada nova entrevista para aplicação de mais dois recordatórios, também relativos aos dois dias precedentes. Os recordatórios alimentares foram realizados com uso de formulário padronizado, com auxílio de álbum fotográfico de porções de alimentos e utensílios de cozinha. ${ }^{12} \mathrm{Os}$ entrevistadores foram treinados para usar perguntas pré-estabelecidas: " - a que horas você se alimentou pela primeira vez, após acordar?”, “- quais os alimentos que você comeu?" e " - bebeu algo?", e 
coletar informações sobre quantidade consumida, tipo de alimento, método de preparo e acréscimo ou não de açúcar ou temperos. O QFA foi aplicado antes dos recordatórios a fim de prevenir a influência destes nas respostas do questionário.

As estimativas de consumo de nutrientes baseadas no QFA foram realizadas com auxílio do aplicativo Excel, através do cálculo: freqüência de consumo x tamanho da porção $\mathrm{x}$ composição nutricional do alimento. Para construção do banco de dados com a composição nutricional dos alimentos foi utilizada a Tabela de Composição de Alimentos: Suporte para Decisão Nutricional/Sonia Tucunduva Philippi. O consumo de nutrientes baseado nos recordatórios alimentares foi estimado com uso do software Virtual Nutri.13,14

Quanto à análise estatística, as comparações entre as variáveis categóricas foram realizadas pelo teste do qui-quadrado, sendo o teste t para amostras independentes utilizado pra comparações de médias.

Inicialmente foi calculada a média dos recordatórios disponíveis para cada gestante (dois recordatórios para as que fizeram uma entrevista e quatro recordatórios para as que fizeram duas entrevistas), sendo esse o valor usado nas demais análises.

As estimativas obtidas pelos dois métodos foram correlacionadas pelo método de Pearson (ou Spearman, quando apropriado). Também foram calculadas correlações ajustadas para consumo energético, isto é, a correlação entre os resíduos padronizados da regressão linear simples entre cada nutriente e o consumo calórico total. O resíduo padronizado de cada regressão linear foi considerado o consumo de nutriente ajustado pelo consumo calórico total. Assim, a correlação ajustada pelo consumo calórico total consiste da correlação entre os resíduos padronizados obtidos pelo QFA e pelo recordatório.

Os dois métodos também foram comparados pela metodologia proposta por Bland e Altman. 15 Para tanto, foram calculados a média da diferença entre os métodos e os limites de concordância (LOAs ou Limits of Agreement), os últimos, usando a fórmula:

LOAs $=$ média diferença $+(1,96 *$ desvio padrão $)$ e média diferença - $(1,96 *$ desvio padrão $)$

A concordância entre os métodos foi examinada também em termos de sua habilidade em classificar as gestantes de acordo com o nível de consumo. Foi calculado o percentual de gestantes classificadas no mesmo quartil e em quartis opostos da distribuição obtida para o nível de consumo segundo os dois métodos. Foi usado o coeficiente de Kappa ponderado, que leva em conta a concordância exata e também classificações próximas. 16
A análise estatística foi realizada com auxílio do Software SPSS versão 13. Em todos os testes foi considerado o nível de significância estatística de 0,05 .

\section{Resultados}

Entre as 161 gestantes selecionadas foram excluídas nove gestantes: uma gestante cuja ecografia realizada no dia da entrevista mostrou óbito fetal, uma gestante que não concluiu a primeira entrevista $\mathrm{e}$ uma hospitalizada no dia anterior à entrevista, que não respondeu ao recordatório. Outras seis gestantes foram excluídas por reportarem consumo de energia conforme QFA acima de $6000 \mathrm{kcal}$. As gestantes excluídas da análise apresentavam idades entre 15 e 36 anos (média 20 anos) e idade gestacional entre nove e 34 semanas (média 20 semanas). As análises aqui descritas, portanto, referem-se a 152 gestantes (82 de Bento Gonçalves e 70 de Porto Alegre).

A Tabela 1 mostra as características sóciodemográficas da amostra. Houve predomínio de gestantes adultas, de baixa renda e no terceiro trimestre da gravidez. Observa-se que as gestantes de Porto Alegre apresentam menor renda e um percentual maior de gestantes solteiras ou separadas.

Quarenta e seis por cento das gestantes responderam às duas entrevistas, sendo esse percentual maior em Bento Gonçalves (58\%) do que em Porto Alegre (31\%). Em relação aos dias a que se referem os recordatórios alimentares, $42 \%$ da amostra total respondeu-os relativos tanto a dias de semana quanto a finais de semana (mistos). Avaliando esse aspecto em separado para cada cidade, observa-se que em Bento Gonçalves a maioria das gestantes respondeu a recordatórios mistos, enquanto em Porto Alegre a maioria das gestantes respondeu a recordatórios relativos apenas a dias de semana. O intervalo de dias entre as duas entrevistas foi maior para gestantes de Porto Alegre (média 18 dias) do que para as de Bento Gonçalves (média 10 dias). Outra diferença observada entre as cidades se refere ao desvio padrão do consumo calórico entre os recordatórios, com média de 547 e 402 e desvio padrão de 388 e 333 em Bento Gonçalves e Porto Alegre, respectivamente.

Agrupando-se as gestantes de acordo com o número de entrevistas realizadas (adesão parcial ou total), não foram observadas diferenças em relação à situação conjugal, escolaridade, faixa etária, renda, recebimento de orientação nutricional e idade gestacional. Em relação à ocupação, as gestantes que realizaram apenas uma entrevista, em geral, não trabalhavam e/ou estudavam, embora essa diferença 
Tabela 1

\begin{tabular}{|c|c|c|c|c|c|c|c|}
\hline \multirow[t]{2}{*}{ Características } & \multicolumn{2}{|c|}{ Total } & \multicolumn{2}{|c|}{ Bento Gonçalves } & \multicolumn{2}{|c|}{ Porto Alegre } & \multirow[t]{2}{*}{$p^{a}$} \\
\hline & $\mathrm{n}$ & $\%$ & $\mathrm{n}$ & $\%$ & $\mathrm{n}$ & $\%$ & \\
\hline \multicolumn{8}{|l|}{ Situação conjugal } \\
\hline Casada/mora com companheiro & 121 & 79,6 & 72 & 87,8 & 49 & 70 & 0,007 \\
\hline Solteira ou separada & 31 & 20,4 & 10 & 12,2 & 21 & 30 & \\
\hline \multicolumn{8}{|l|}{ Ocupação da gestante } \\
\hline Não estuda e nem trabalha & 85 & 55,9 & 49 & 59,8 & 36 & 51,4 & 0,193 \\
\hline Estuda e/ou trabalha & 67 & 44,1 & 33 & 40,2 & 34 & 48,6 & \\
\hline \multicolumn{8}{|l|}{ Escolaridade (anos) } \\
\hline Até 4 & 14 & 9,2 & 10 & 12,2 & 4 & 5,7 & 0,348 \\
\hline 5 a 8 & 76 & 50 & 41 & 50 & 35 & 50 & \\
\hline Mais de 8 & 62 & 40,8 & 31 & 37,8 & 31 & 44,3 & \\
\hline \multicolumn{8}{|l|}{ Orientação nutricional } \\
\hline $\operatorname{sim}$ & 39 & 25,7 & 26 & 31,7 & 13 & 18,6 & 0,065 \\
\hline Não & 113 & 74,3 & 56 & 68,3 & 57 & 81,4 & \\
\hline \multicolumn{8}{|l|}{ Faixa etária } \\
\hline Adolescente (15 a 18 anos) & 30 & 19,7 & 18 & 22 & 12 & 17,1 & 0,458 \\
\hline Adulta (mais de 18 a 42 anos) & 122 & 80,3 & 64 & 78 & 58 & 82,9 & \\
\hline \multicolumn{8}{|l|}{ Idade Gestacional (trimestre) } \\
\hline Primeiro & 12 & 7,9 & 5 & 6,1 & 7 & 10 & 0,336 \\
\hline Segundo & 61 & 40,1 & 37 & 45,1 & 24 & 34,3 & \\
\hline Terceiro & 79 & 52 & 40 & 48,8 & 39 & 55,7 & \\
\hline \multicolumn{8}{|l|}{ Número de pessoas na casa } \\
\hline Até 3 pessoas & 82 & 53,9 & 46 & 56,1 & 36 & 51,4 & 0,054 \\
\hline 4 a 6 pessoas & 62 & 40,8 & 35 & 42,7 & 27 & 38,6 & \\
\hline 7 ou mais pessoas & 8 & 5,3 & 1 & 1,2 & 7 & 10 & \\
\hline \multicolumn{8}{|l|}{ Renda per capita } \\
\hline Até $\mathrm{R} \$ 150,00$ & 32 & 21,1 & 8 & 9,8 & 24 & 34,3 & $<0,001$ \\
\hline$R \$ 150,00$ a $R \$ 300,00$ & 107 & 70,4 & 69 & 84,1 & 38 & 54,3 & \\
\hline Acima de $R \$ 300,00$ & 13 & 8,6 & 5 & 6,1 & 8 & 11,4 & \\
\hline
\end{tabular}

$a=$ teste qui-quadrado

tenha alcançado valores limítrofes de significância estatística $(p=0,05)$ apenas em Porto Alegre.

A Tabela 2 apresenta medidas comparativas dos dois métodos. O coeficiente de correlação variou de 0,01 (Vitamina E) a 0,43 (Vitamina C). Já o coeficiente de correlação ajustado para energia variou de 0,10 (colesterol) a 0,50 (Vitamina C). Correlações maiores (acima de 0,4) foram observadas para fibras, vitamina $\mathrm{C}$, ácido fólico, cálcio e potássio, sendo essas semelhantes entre as duas cidades. A diferença média e limites de concordância (LOAS) entre os dois métodos também são apresentados na Tabela 2. Observa-se que gestantes de Porto Alegre apresentaram limites de concordância mais amplos, exceto para colesterol, vitamina B12, vitamina B2, sódio, cálcio, zinco e cobre.

A Tabela 3 mostra a concordância entre os métodos na classificação das gestantes por quartis de consumo na amostra total e para cada cidade. $\mathrm{O}$ valor de Kappa ponderado variou de 0,39 (vitamina C) a 0,06 (cobre) na amostra total. Entre gestantes de Bento Gonçalves variou de 0,62 (vitamina B1) a 0,18 (vitamina A), enquanto para gestantes de Porto Alegre os resultados ficaram entre 0,51 (cálcio) a 0,01 (colesterol).

Na Tabela 4 é apresentada a concordância entre os métodos de acordo com o nível de escolaridade da gestante. Observou-se maior concordância 


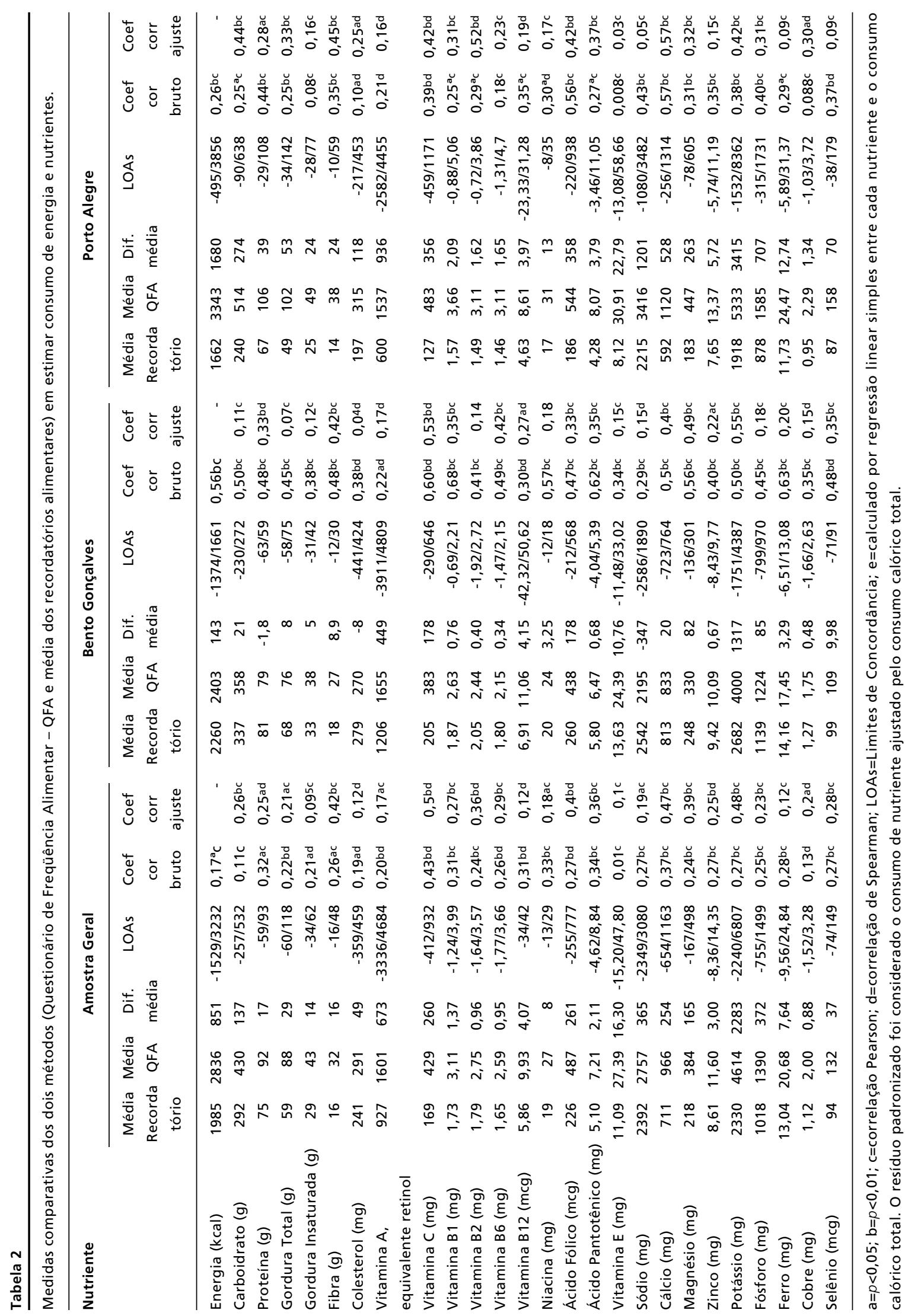


Tabela 3

Número de gestantes classificadas no mesmo quartil e no quartil oposto pelos dois métodos (Questionário de Freqüência Alimentar - QFA e média dos recordatórios alimentares).

\begin{tabular}{|c|c|c|c|c|c|c|c|c|c|c|c|c|c|c|c|}
\hline \multirow[t]{3}{*}{ Nutriente } & \multicolumn{5}{|c|}{ Geral $n=152$} & \multicolumn{5}{|c|}{ Bento Gonçalves $n=82$} & \multicolumn{5}{|c|}{ Porto Alegre $n=70$} \\
\hline & \multicolumn{2}{|c|}{$\begin{array}{l}\text { Mesmo } \\
\text { quartil }\end{array}$} & \multicolumn{2}{|c|}{$\begin{array}{l}\text { Quartil } \\
\text { oposto }\end{array}$} & \multirow[t]{2}{*}{ Kappa } & \multicolumn{2}{|c|}{$\begin{array}{l}\text { Mesmo } \\
\text { quartil }\end{array}$} & \multicolumn{2}{|c|}{$\begin{array}{l}\text { Quartil } \\
\text { oposto }\end{array}$} & \multirow[t]{2}{*}{ Kappa } & \multicolumn{2}{|c|}{$\begin{array}{l}\text { Mesmo } \\
\text { quartil }\end{array}$} & \multicolumn{2}{|c|}{$\begin{array}{l}\text { Quartil } \\
\text { oposto }\end{array}$} & \multirow[t]{2}{*}{ Kappa } \\
\hline & $\mathrm{n}$ & $\%$ & $\mathrm{n}$ & $\%$ & & $\mathrm{n}$ & $\%$ & $\mathrm{n}$ & $\%$ & & $\mathrm{n}$ & $\%$ & $\mathrm{n}$ & $\%$ & \\
\hline Energia (kcal) & 46 & 30 & 14 & 9 & 0,16 & 38 & 46 & 1 & 1 & 0,53 & 22 & 27 & 3 & 4 & 0,19 \\
\hline Carboidrato (g) & 40 & 26 & 15 & 10 & 0,11 & 39 & 48 & 4 & 5 & 0,46 & 13 & 16 & 3 & 4 & 0,23 \\
\hline Proteína (g) & 49 & 32 & 13 & 9 & 0,23 & 36 & 44 & 4 & 5 & 0,49 & 25 & 30 & 5 & 6 & 0,20 \\
\hline Gordura Total (g) & 46 & 30 & 15 & 10 & 0,20 & 30 & 37 & 5 & 6 & 0,36 & 25 & 30 & 4 & 5 & 0,33 \\
\hline $\begin{array}{l}\text { Gordura } \\
\text { Insaturada (g) }\end{array}$ & 44 & 29 & 11 & 7 & 0,20 & 30 & 37 & 4 & 5 & 0,37 & 18 & 22 & 7 & 9 & 0,16 \\
\hline Fibra (g) & 44 & 29 & 15 & 10 & 0,10 & 31 & 38 & 2 & 2 & 0,41 & 25 & 30 & 7 & 9 & 0,18 \\
\hline Colesterol (mg) & 47 & 31 & 12 & 8 & 0,21 & 29 & 35 & 2 & 2 & 0,4 & 20 & 24 & 9 & 11 & 0,01 \\
\hline $\begin{array}{l}\text { Vitamina } A \text {, } \\
\text { equivalente retinol }\end{array}$ & 43 & 28 & 16 & 11 & 0,16 & 29 & 35 & 9 & 11 & 0,18 & 24 & 29 & 4 & 5 & 0,19 \\
\hline Vitamina C (mg) & 59 & 39 & 7 & 5 & 0,39 & 38 & 46 & 4 & 5 & 0,5 & 27 & 33 & 3 & 4 & 0,38 \\
\hline Vitamina B1 (mg) & 47 & 31 & 7 & 5 & 0,30 & 38 & 46 & 1 & 1 & 0,62 & 20 & 24 & 4 & 5 & 0,24 \\
\hline Vitamina B2 (mg) & 40 & 26 & 10 & 7 & 0,23 & 31 & 38 & 1 & 1 & 0,51 & 24 & 29 & 2 & 2 & 0,32 \\
\hline Vitamina B6 (mg) & 48 & 31 & 13 & 9 & 0,25 & 32 & 39 & 2 & 2 & 0,49 & 21 & 26 & 7 & 9 & 0,16 \\
\hline Vitamina B12 (mcg) & 55 & 36 & 9 & 6 & 0,31 & 31 & 38 & 5 & 6 & 0,26 & 25 & 30 & 4 & 5 & 0,36 \\
\hline Niacina (mg) & 47 & 31 & 16 & 11 & 0,24 & 35 & 43 & 3 & 4 & 0,48 & 24 & 29 & 5 & 6 & 0,13 \\
\hline Ácido Fólico (mcg) & 53 & 35 & 12 & 8 & 0,26 & 29 & 35 & 1 & 1 & 0,41 & 24 & 29 & 5 & 6 & 0,29 \\
\hline $\begin{array}{l}\text { Ácido } \\
\text { Pantotênico (mg) }\end{array}$ & 52 & 34 & 5 & 3 & 0,38 & 32 & 39 & 3 & 4 & 0,51 & 22 & 27 & 4 & 5 & 0,32 \\
\hline Vitamina E (mg) & 38 & 25 & 17 & 11 & 0,09 & 24 & 29 & 3 & 4 & 0,35 & 17 & 21 & 8 & 10 & $-0,02$ \\
\hline Sódio (mg) & 46 & 30 & 11 & 7 & 0,24 & 25 & 30 & 5 & 6 & 0,35 & 28 & 34 & 1 & 1 & 0,43 \\
\hline Cálcio (mg) & 53 & 35 & 6 & 4 & 0,37 & 27 & 33 & 2 & 2 & 0,45 & 34 & 41 & 3 & 4 & 0,51 \\
\hline Magnésio (mg) & 41 & 27) & 12 & 8 & 0,21 & 40 & 49 & 2 & 2 & 0,5 & 25 & 30 & 4 & 5 & 0,22 \\
\hline Zinco (mg) & 43 & 28 & 11 & 7 & 0,25 & 31 & 38 & 3 & 4 & 0,49 & 24 & 29 & 5 & 6 & 0,25 \\
\hline Potássio (mg) & 40 & 26 & 10 & 7 & 0,23 & 34 & 41 & 1 & 1 & 0,54 & 26 & 32 & 6 & 7 & 0,25 \\
\hline Fósforo (mg) & 45 & 30 & 13 & 9 & 0,22 & 31 & 38 & 2 & 2 & 0,41 & 22 & 27 & 4 & 5 & 0,29 \\
\hline Ferro (mg) & 51 & 33 & 10 & 7 & 0,31 & 33 & 40 & 0 & 0 & 0,56 & 24 & 29 & 4 & 5 & 0,26 \\
\hline Cobre (mg) & 42 & 28 & 19 & 12 & 0,06 & 29 & 35 & 2 & 2 & 0,4 & 21 & 26 & 8 & 10 & 0,05 \\
\hline Selênio (mcg) & 49 & 32 & 8 & 5 & 0,28 & 31 & 38 & 2 & 2 & 0,47 & 22 & 27 & 1 & 1 & 0,29 \\
\hline
\end{tabular}


Número de gestantes classificadas no mesmo quartil e no quartil oposto pelos dois métodos (Questionário de Freqüência Alimentar - QFA e média dos recordatórios alimentares), estratificando conforme escolaridade.

\begin{tabular}{|c|c|c|c|c|c|c|c|c|c|c|}
\hline \multirow[t]{3}{*}{ Nutriente } & \multicolumn{5}{|c|}{ Escolaridade até 7 anos $=61$} & \multicolumn{5}{|c|}{ Escolaridade 8 ou mais anos $=91$} \\
\hline & \multicolumn{2}{|c|}{$\begin{array}{l}\text { Mesmo } \\
\text { quartil }\end{array}$} & \multicolumn{2}{|c|}{$\begin{array}{l}\text { Quartil } \\
\text { oposto }\end{array}$} & \multirow[t]{2}{*}{ Kappa } & \multicolumn{2}{|c|}{$\begin{array}{l}\text { Mesmo } \\
\text { quartil }\end{array}$} & \multicolumn{2}{|c|}{$\begin{array}{l}\text { Quartil } \\
\text { oposto }\end{array}$} & \multirow[t]{2}{*}{ Kappa } \\
\hline & $\mathrm{n}$ & $\%$ & $\mathrm{n}$ & $\%$ & & $\mathrm{n}$ & $\%$ & $\mathrm{n}$ & $\%$ & \\
\hline Energia (kcal) & 17 & 27,8 & 7 & 11,5 & 0,06 & 30 & 32,9 & 8 & 8,8 & 0,19 \\
\hline Carboidrato (g) & 21 & 34,4 & 7 & 11,5 & 0,04 & 19 & 20,8 & 9 & 9,9 & 0,12 \\
\hline Proteína (g) & 13 & 21,3 & 6 & 9,8 & 0,08 & 37 & 40,6 & 7 & 7,7 & 0,33 \\
\hline Gordura Total (g) & 17 & 27,9 & 6 & 9,8 & 0,15 & 30 & 32,9 & 8 & 8,8 & 0,25 \\
\hline Gordura Insaturada (g) & 18 & 29,5 & 6 & 9,8 & 0,14 & 25 & 27,5 & 5 & 5,5 & 0,24 \\
\hline Fibra (g) & 17 & 27,8 & 4 & 6,5 & 0,10 & 25 & 27,5 & 7 & 7,7 & 0,14 \\
\hline Colesterol (mg) & 20 & 32,8 & 4 & 6,6 & 0,22 & 29 & 31,9 & 9 & 9,9 & 0,19 \\
\hline Vitamina $A$, equivalente retinol & 13 & 21,3 & 7 & 11,5 & 0,11 & 31 & 34 & 9 & 9,9 & 0,23 \\
\hline Vitamina C (mg) & 20 & 32,8 & 5 & 8,2 & 0,16 & 37 & 40,6 & 3 & 3,3 & 0,49 \\
\hline Vitamina B1 (mg) & 18 & 29,5 & 3 & 4,9 & 0,26 & 27 & 29,7 & 6 & 6,6 & 0,29 \\
\hline Vitamina B2 (mg) & 15 & 24,6 & 5 & 8,2 & 0,15 & 28 & 30,8 & 5 & 5,5 & 0,29 \\
\hline Vitamina B6 (mg) & 18 & 29,5 & 5 & 8,2 & 0,19 & 33 & 36,3 & 8 & 8,8 & 0,30 \\
\hline Vitamina B12 (mcg) & 27 & 44,3 & 4 & 6,6 & 0,32 & 30 & 32,9 & 5 & 5,5 & 0,30 \\
\hline Niacina (mg) & 18 & 29,5 & 6 & 9,8 & 0,18 & 32 & 35,0 & 9 & 9,9 & 0,27 \\
\hline Ácido Fólico (mcg) & 15 & 24,6 & 6 & 9,8 & 0,10 & 32 & 35,1 & 6 & 6,6 & 0,30 \\
\hline Ácido Pantotênico (mg) & 18 & 29,5 & 5 & 8,2 & 0,27 & 32 & 35,2 & 3 & 3,3 & 0,43 \\
\hline Vitamina $\mathrm{E}(\mathrm{mg})$ & 14 & 22,9 & 8 & 14,7 & $-0,01$ & 24 & 26,4 & 8 & 8,8 & 0,14 \\
\hline Sódio (mg) & 17 & 27,8 & 4 & 6,5 & 0,26 & 29 & 31,8 & 8 & 8,8 & 0,20 \\
\hline Cálcio (mg) & 18 & 29,5 & 2 & 3,3 & 0,39 & 34 & 37,4 & 3 & 3,3 & 0,36 \\
\hline Magnésio (mg) & 15 & 24,6 & 6 & 9,8 & 0,10 & 25 & 27,5 & 4 & 4,4 & 0,30 \\
\hline Zinco (mg) & 19 & 31,1 & 3 & 4,9 & 0,16 & 24 & 26,4 & 6 & 6,6 & 0,32 \\
\hline Potássio (mg) & 11 & 18,0 & 6 & 9,8 & 0,03 & 26 & 28,6 & 3 & 3,3 & 0,33 \\
\hline Fósforo (mg) & 14 & 22,9 & 6 & 9,8 & 0,11 & 31 & 34,0 & 7 & 7,7 & 0,30 \\
\hline Ferro (mg) & 17 & 27,8 & 4 & 6,6 & 0,22 & 30 & 32,9 & 7 & 7,7 & 0,31 \\
\hline Cobre (mg) & 16 & 26,2 & 10 & 16,4 & $-0,13$ & 26 & 28,6 & 8 & 8,8 & 0,17 \\
\hline Selênio (mcg) & 20 & 32,8 & 2 & 3,3 & 0,32 & 28 & 30,8 & 6 & 6,6 & 0,25 \\
\hline
\end{tabular}

(segundo valor de kappa) para gestantes com oito ou mais anos de escolaridade, exceto para colesterol, vitamina B12, sódio, cálcio e selênio.

\section{Discussão}

Os resultados desse estudo de validação relativa comparam as estimativas de consumo de energia e 25 nutrientes provenientes do QFA elaborado por Sichieri e Everhart 10 com a média de dois ou quatro recordatórios alimentares, em um grupo populacional específico - gestantes. No primeiro estudo de validação foi avaliado o consumo de carboidrato, proteína, gordura, vitamina A, Vitamina $\mathrm{C}$, cálcio e ferro, enquanto no presente estudo outros nutrientes, como ácido fólico, cobre, zinco, selênio, vitaminas do complexo $\mathrm{B}$, potássio e fibras, foram incluídos. Esse questionário vem sendo utilizado em diversos estudos epidemiológicos, em gestantes, adolescentes e adultos. ${ }^{17,18}$

A metodologia proposta por Bland e Altman, ${ }^{15}$ recomendada para uso em estudos de validação de questionários de freqüência alimentar, foi aplicada nesse trabalho. Porém, tem sido pouco utilizada no Brasil, dificultando comparações com os resultados obtidos em outras validações.

Recente estudo, realizado no Reino Unido, mostrou a validade relativa de um QFA desenvolvido para uso em gestantes, para fins de triagem nutricional, apresentando os gráficos propostos por Bland e Altman. 15 Nutrientes como gordura mono e poliin- 
saturada, fibras, proteínas e álcool apresentaram limites de concordância maiores. 19

No presente estudo, os nutrientes com limites de concordância mais amplos foram vitamina $\mathrm{B} 12$, vitamina $\mathrm{E}$, vitamina $\mathrm{C}$ e vitamina $\mathrm{A}$, mostrando maior discordância entre os dois métodos (QFA e recordatórios) para esses nutrientes. O QFA, em média, superestimou o consumo de energia e nutrientes, exceto para proteína, colesterol e sódio, entre gestantes de Bento Gonçalves.

Outros estudos, também realizados em gestantes, mostraram estimativas mais elevadas obtidas de questionários de freqüência alimentar. Forsythe e Gage $^{20}$ realizaram estudo de validação com 80 gestantes com alta escolaridade e obtiveram médias de consumo baseadas no QFA maiores do que em recordatórios alimentares, exceto para sódio. Erkkola et al.21 observaram que estimativas de consumo de um QFA composto por 181 itens foram 30 a $40 \%$ maiores do que a média de registros alimentares, sugerindo a hipótese de que gestantes possam superestimar o consumo mais do que outras pessoas. O desejo de aceitação social e idealização de alimentação saudável, possivelmente maiores na gestação, podem explicar este fenômeno. 20,21

Os resultados desse estudo, quanto à concordância entre o QFA e o método de referência, para classificação dos indivíduos em níveis de consumo foram similares aos encontrados em outros estudos, com gestantes e outros grupos populacionais. Cardoso et al.,22 Henn 23 e Robinson et al. 24 identificaram que em torno de 30 a $40 \%$ dos indivíduos foram classificados no mesmo quartil de consumo tanto pelo QFA quanto pelo método de referência. No presente estudo, o percentual de concordância variou de $25 \%$ para consumo de vitamina E a $35 \%$ para consumo de ácido fólico e cálcio. Segundo nosso conhecimento, esse é o primeiro estudo avaliando a habilidade do QFA desenvolvido por Sichieri e Everhart 10 em classificar os indivíduos conforme quartis de consumo.

No estudo de validação conduzido por Sichieri e Everhart ${ }^{10}$ são apresentados apenas os coeficientes de correlação brutos, os quais são maiores do que os observados neste estudo para energia, proteína, carboidrato, gordura e ferro, menores para vitamina $\mathrm{C}$ e similares para vitamina A e cálcio. 10

No presente estudo observaram-se coeficientes de correlação abaixo do esperado, porém similares aos obtidos em outros estudos com gestantes, conduzidos por Mouratidou et al.19 e Forsythe e Gage. 20 Segundo Baer et al.,25 durante a gestação a alimentação é menos estável, o que poderia levar a diminuição nas correlações entre dois métodos de inquérito alimentar. Além disso, gestantes estão mais expostas a mensagens sobre alimentação, fator que sabidamente dificulta a avaliação da ingestão alimentar. $19,20,25$

As baixas correlações encontradas, tanto no presente estudo quanto em outros realizados com gestantes ou não, para nutrientes como colesterol e vitamina A, vitamina E e vitamina B12, podem estar relacionadas à concentração desses nutrientes nos alimentos, pois as correlações relatadas na literatura tendem a ser menores para nutrientes encontrados em grande quantidade em um número limitado de alimentos, principalmente quando o recordatório não é realizado no dia em que esses alimentos tenham sido consumidos. $22,25,26$

Correlações maiores (coeficiente de correlação ajustado acima de 0,4 ) foram observadas para fibras, vitamina $\mathrm{C}$, ácido fólico, cálcio e potássio. As correlações para esses nutrientes foram semelhantes quando avaliadas a amostra total e em separado por cidade.

Para outros nutrientes, como carboidrato, gordura, vitamina E e sódio, foram observadas diferenças nos coeficientes de correlação entre as duas cidades. Diferenças dessa natureza também foram observadas por Sichieri e Everhart,10 entre professores e funcionários de universidade (sendo maior entre professores, exceto para cálcio e proteína) e por Baer et al., 25 entre gestantes de etnias diferentes e com rendas diferentes, sendo que as correlações foram maiores para gestantes caucasianas e para gestantes de menor condição econômica.10,25

É bem reconhecido que o QFA pode ter desempenho diferente em populações diferentes, o que levou à recomendação de que ele seja validado para cada população. ${ }^{9}$ As diferenças entre cidades, observadas nesse estudo, mostram que essa recomendação é consistente.

Estudar as características das gestantes que podem predispor a erro no relato do consumo é um aspecto importante, que merece atenção em outros estudos. A menor concordância entre QFA e recordatórios observada entre gestantes com diferente escolaridade sugere que essa pode desempenhar papel importante, influenciando o relato do consumo através de questionários de freqüência alimentar. Nesse estudo, outra possibilidade a ser considerada é de que as gestantes de Porto Alegre, por serem terem uma condição socioeconômica menos privilegiada, possam ter se constrangido mais ao relatar seu consumo, superestimando-o. Situação semelhante foi descrita por Olinto et al.,27 ao observar que mães de crianças desnutridas, de baixa condição socioeconômica, superestimavam o consumo de energia, 
proteína e lipídeos, quando aplicado o recordatório de $24 \mathrm{hs} .27$

Entre as limitações desse estudo, inclui-se o curto intervalo de tempo para coleta de dados (três meses) e a ausência de medidas que permitam quantificar o viés do entrevistador e do entrevistado. Também se podem citar as perdas relativas à segunda entrevista, na sua maioria justificadas por recomendação médica para repouso, nascimento do bebê antes da data provável e o fato de a gestante trabalhar.

O fato de que as gestantes de Porto Alegre, na sua maioria, responderam recordatórios relativos apenas a dias de semana, possivelmente por terem realizado apenas uma entrevista, também representa uma limitação desse trabalho. Sabe-se que o consumo alimentar varia de um dia para outro, sendo maior nos finais de semana. Sendo assim, recordatórios relativos apenas a dias de semana não representam a dieta usual do indivíduo, tanto quanto recordatórios que incluam sábados ou domingos. O desvio padrão entre os recordatórios alimentares mostrou-se maior entre as gestantes de Bento Gonçalves, independente do número de entrevista realizadas, demonstrando a possível influência da diferença nos dias da semana em que foi realizada a entrevista nos resultados do trabalho. Pode-se supor que esses dois fatores, a perda de gestantes para realização da segunda entrevista e a diferença no dia da semana em que os recordatórios foram aplicados, tendem a diminuir as correlações entre o QFA e os recordatórios e também aumentar as diferenças entre cidades.

Considerando que o estudo incluiu somente gestantes em atendimento pelo SUS, é importante, também, salientar que o questionário pode não ter o mesmo desempenho se aplicado a todas as gestantes das duas cidades.

\section{Referências}

1. Neggers Y, Goldenberg RL. Some thoughts on body mass index, micronutrient intakes and pregnancy outcome. J Nutr. 2003; 133 (Suppl 2): S1737-40.

2. Kaiser LL, Allen L. Position of the American Dietetic Association: nutrition and lifestyle for a healthy pregnancy outcome. J Am Diet Assoc. 2002; 102: 1479-90.

3. Fonseca VM, Sichieri R, Basilio L, Ribeiro LVC. Consumo de folato em gestantes de um hospital público do Rio de Janeiro. Rev Bras Epidemiol. 2003; 6: 319-27.

4. Azevedo DA, Sampaio HAC. Consumo alimentar de gestantes adolescentes atendidas em serviço de assistência pré-natal. Rev Nutr. 2003; 16: 273-80.
Devido à natureza complexa da dieta dos indivíduos e à substancial variabilidade da alimentação ao longo do tempo, os erros de medida são inevitáveis. ${ }^{28}$ Considerando que o QFA é um método de baixo custo e fácil análise, sugere-se que maiores esforços sejam feitos a fim de melhorar seu desempenho. Entre as alternativas possíveis, destaca-se a avaliação das porções oferecidas para determinar quantidade de consumo (Ex: para biscoito, usar como medida "unidade" e não "pacote"). Álbuns fotográficos desenvolvidos com os alimentos do questionário e suas respectivas quantidades de referência também podem ser úteis.

Outra opção para melhorar a validade do instrumento é o uso de questões do tipo "cross-check", perguntando, por exemplo, "quantas porções de fruta você come por semana?", tornando possível estabelecer fatores de correção para a freqüência de consumo. Essa abordagem é especialmente útil para evitar superestimação no consumo de frutas e verduras. 29

\section{Conclusões}

O Questionário de Freqüência Alimentar mostrou-se uma ferramenta exeqüível para avaliar o consumo alimentar de gestantes. Quando comparado ao recordatório, o QFA superestima o consumo e mostra, em geral, baixas correlações, o que precisa ser considerado na interpretação dos achados. Quando usado para classificar indivíduos conforme nível de consumo, o QFA mostrou capacidade semelhante a outros questionários desenvolvidos no Brasil. Estratégias para melhorar seu desempenho precisam ser investigadas, incluindo a identificação dos fatores que podem afetar sua validade.

5. Barros DC, Pereira RA, Gama SGN, Leal MC. O consumo alimentar de gestantes adolescentes no Município do Rio de Janeiro. Cad Saúde Pública. 2004; 20 (Suppl. 1):S121-9.

6. Zulkifli SN, Stella MY. The food frequency method for dietary assessment. J Am Diet Assoc. 1992; 92: 681-5.

7. Fisberg RM, Slater B, Marchioni DML, Martini LA. Inquéritos alimentares: métodos e bases científicas. Barueri: Manole; 2005. v.1.

8. Slater B, Philippi ST, Marchioni DM, Fisberg RM. Validação de Questionário de Freqüência Alimentar - QFA: considerações metodológicas. Rev Bras Epidemiol. 2003; 6: 200-8. 
9. Cade J, Thompson R, Burley V, Warm D. Development, validation and utilisation of food-frequency questionnaires - a review. Public Health Nutr. 2002; 5: 567-87.

10. Sichieri R, Everhart JE. Validity of a brazilian food frequency questionary against dietary recalls and estimated energy intake. Nutrit Res. 1998; 18: 1649-59.

11. Sichieri R. Epidemiologia da obesidade. Rio de Janeiro: Ed UERJ; 1998. v.1.

12. Zaboto $\mathrm{CB}$. Registro fotográfico para inquéritos dietéticos. Goiânia: Metha; 1996.

13. Philippi ST. Tabela de composição de alimentos: suporte para decisão nutricional. 2. ed. São Paulo: Coronário; 2002.

14. Philippi ST, Szarfarc SC, Laterza AR. Virtual Nutri [software]. São Paulo: Departamento de Nutrição da Faculdade de Saúde Pública da Universidade de São Paulo; 1996.

15. Bland JM, Altman DG. Statistical methods for assessing agreement between two methods of clinical measurement. Lancet. 1986; 1: 307-10.

16. Cohen J. Weighted kappa: nominal scale agreement with provision for scaled disagreement or partial credit. J Psychol Bull. 1968; 70: 213-20.

17. Castro MB, Kac G, Sichieri R. Dietary patterns among postpartum women treated at a municipal health center in Rio de Janeiro, Brazil. Cad Saúde Pública. 2006; 22: 1159-70.

18. Sichieri R. Dietary patterns and their associations with obesity in the Brazilian city of Rio de Janeiro. Obes Res. 2002; 10: 42-8.

19. Mouratidou T, Ford F, Fraser RB. Validation of a foodfrequency questionnaire for use in pregnancy. Public Health Nutr. 2006; 9: 515-22.

20. Forsythe HE, Gage B. Use of a multicultural foodfrequency questionnaire with pregnant and lactating women. Am J Clin Nutr. 1994; 59 (Suppl 1): S203-6.

21. Erkkola M, Karppinen M, Javanainen J, Knip M, Virtanen SM. Validity and reproducibility of a food frequency questionnaire for pregnant Finnish women. Am J Epidemiol. 2001; 154: 466-76.

Recebido em 6 de março de 2007

Versão final apresentada em 3 de novembro de 2007

Aprovado em 10 de novembro de 2008
22. Cardoso MA, Kida AA, Tomita LY, Stocco PR. Reproducibility and validity of a food frequency questionnaire among women of Japanese ancestry living in Brazil. Nutrit Res. 2001; 21: 725-33.

23. Henn RL. Padrão alimentar e excesso de peso em uma população adulta da cidade de Porto Alegre, RS. [tese]. Porto Alegre: Programa de Pós-Graduação em Epidemiologia da Universidade Federal do Rio Grande do Sul; 2006.

24. Robinson S, Godfrey K, Osmond C, Cox V, Barker D. Evaluation of a food frequency questionnaire used to assess nutrient intakes in pregnant women. Eur J Clin Nutr. 1996; 50: 302-8.

25. Baer HJ, Blun RE, Rockett HRH, Leppert J, Gardner JD, Suitor CW, Colditz GA. Use of a food frequency questionnaire in American Indian and Caucasian pregnant women: a validation study. BMC Public Health. 2005; 5: 135. [periódico online]. Available from: http://www. biomedcentral.com/1471-2458/5/135

26. Wei EK, Gardner J, Field AE, Rosner BA, Colditz GA, Suitor CW. Validity of a food frequency questionnaire in assessing nutrient intakes of low-income pregnant women. Matern Child Health J. 1999; 3: 241-6.

27. Olinto MT, Victora CG, Barros FC, Gigante DP. Twentyfour-hour recall overestimates the dietary intake of malnourished children. J Nutr. 1995; 125: 880-4.

28. Willett W. Invited commentary: a further look at dietary questionnaire validation [discussion]. Am J Epidemiol. 2001; 154:1100-2; 1105-6.

29. Calvert C, Cade J, Barrett JH, Woodhouse A. Using crosscheck questions to address the problem of mis-reporting of specific food groups on Food Frequency Questionnaires. UKWCS Steering Group. United Kingdom Women's Cohort Study Steering Group. Eur J Clin Nutr. 1997; 51: 708-12. 